\title{
Initial clinical experience with frameless optically guided stereotactic radiosurgery/radiotherapy in pediatric patients
}

\author{
Sassan Keshavarzi • Hal Meltzer • Sharona Ben-Haim • \\ Charles Benjamin Newman • Joshua D Lawson • \\ Michael L. Levy • Kevin Murphy
}

Received: 31 January 2009/Published online: 27 March 2009

(C) The Author(s) 2009. This article is published with open access at Springerlink.com

\begin{abstract}
Objective The objective of this study is to report our initial experience treating pediatric patients with central nervous system tumors using a frameless, optically guided linear accelerator.

Materials and methods Pediatric patients were selected for treatment after evaluation by a multidisciplinary neurooncology team including neurosurgery, neurology, pathology, oncology, and radiation oncology. Prior to treatment, all patients underwent treatment planning using magnetic resonance imaging (MRI) and treatment simulation on a standard computed tomography scanner (CT). For CT simulation, patients were fitted with a customized plastic face mask with a bite block attached to an optical array with four reflective markers. After ensuring adequate reproducibility, these markers were tracked during treatment by an infra-red camera. All treatments were delivered on a Varian
\end{abstract}

Dr. Kevin Murphy and the Department of Radiation Oncology at UCSD receives monetary support from Varian corporation.

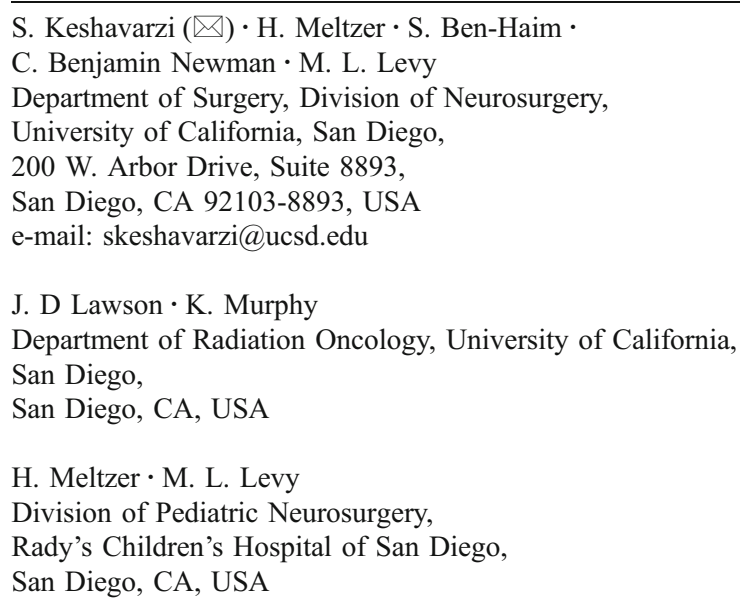

Trilogy linear accelerator. The follow-up period ranges from 1-18 months, with a median follow-up of 6 months. Results Nine patients, ages ranging from 12 to 19 years old (median age 15 years old), with a variety of tumors have been treated. Patients were treated for juvenile pilocytic astrocytoma (JPA; $n=2)$, pontine low-grade astrocytoma $(n=1)$, pituitary adenoma $(n=3)$, metastatic medulloblastoma $(n=1)$, acoustic neuroma $(n=1)$, and pineocytoma $(n=$ 1 ). We followed patients for a median of 12 months (range 3-18 months) with no in-field failures and were able to obtain encouraging toxicity profiles.

Conclusion Frameless stereotactic optically guided radiosurgery and radiotherapy provides a feasible and accurate tool to treat a number of benign and malignant tumors in children with minimal treatment-related morbidity.

Keywords Brain tumor · Pediatric · Radiosurgery · Frameless

\section{Introduction}

An aggressive multidisciplinary approach to the treatment of CNS tumors frequently includes chemotherapy, radiation, and surgery $[1,2]$. Each of these modalities carries a certain amount of morbidity which must be weighed against its expected benefit. Radiation continues to play a vital role in the curative and palliative management of intracranial tumors in children but is frequently limited due to patient age and associated toxicity. Children are particularly sensitive to the potential toxicities of radiation including cognitive impairment, skeletal growth delay/arrest, and secondary malignancy [3].

Stereotactic radiosurgical protocols utilizing steep dose gradients and the use of multiple intersecting conical beams 
[4-6] to deliver a single treatment dose with great precision to a small treatment area have enhanced the safety by which radiation can be delivered. Additional variants of this technology include multi-leaf collimators (MLC), intensity-modulated radiotherapy (IMRT) and fractionated stereotactic radiotherapy (FSR) $[4,6,7]$. One challenge of FSR in children, especially hyperfractionated therapy with more than 18-25 fractions of radiation, has been to develop a frameless system that allows for multiple treatment sessions while maintaining treatment accuracy. In this regard, there has been recent progress in frameless SRS technology [8-10]. At the Moores Cancer Center of the University of California, San Diego, children and adolescents are initially fitted with a thick thermoplastic mask and a bite block. The mask also incorporates frameless stereotactic fiducial spheres which allows for positioning with the accuracy of frame-based radiosurgery while avoiding rigid head-frames. This technique allows for the avoidance of rigid pin head fixation during therapy. Following is our initial experience using this frameless technique in nine pediatric patients with CNS tumors.

\section{Materials and methods}

Selected patients underwent a treatment planning MRI with a radiosurgery protocol (fast $3 \mathrm{D}$ volumetric T1 scan $512 \times$ $512,1.5 \mathrm{~mm}$ thickness with a $26 \mathrm{~cm}$ FOV and no tilt FOV). A customized immobilization device was fabricated for each patient consisting of a headrest, bite block, and mask. The headrest was molded using Accuform (Med-Tec) with the patient's head placed in the neutral position. To develop the bite block, a dental mold made of dental cement was placed into the bite tray and an impression of the patient's upper teeth was obtained. After allowing the mold to set, an array of four reflective markers was attached to the bite block, placed in one plane $10-15^{\circ}$ from the horizontal. Using the bite block as an attachment to the patient's teeth, these markers are used as surrogates for patient positioning. Patients are taken to the treatment room where the optical guidance cameras are vaulted to the ceiling. Each patient wears a helmet with a set of test fiducials attached. The patient inserts and removes the bite block (with the markers attached) ten times and each time the relative positioning error is measured. Variation in positioning of $<0.75 \mathrm{~mm}$, as measured by the optical guidance camera, was considered acceptable. Once reproducibility has been assessed, patients were taken to the CT simulator. Here, the thermoplastic mask was placed over the patient's head and molded to their face. With the mask and bite block in place, the patients underwent simulation CT (FOV $35 \mathrm{~cm}, 1.25 \mathrm{~mm}$ slice thickness, with no contrast), with minimization of metal artifact (Fig. 1).

Following CT registration, the FastPlan software detects, registers, and verifies the fiducial markers (Fig. 2). The CT and planning MR images were then fused (both manually and automatically) using a rigid auto-registration tool with mutual information metrics (Fig. 3). Varian's Eclipse treatment planning system was used and target volume and normal organ-at-risk (OAR) volumes were then contoured by the treating radiation oncologist and neurosurgeon. Margins were added at the discretion of the treating radiation oncologist, and were typically $1-3 \mathrm{~mm}$. No margin was added to any contoured OAR. After meticulous target volume contouring, treatment planning commenced. Isocenter placement was either automatic or manual. The two collimators utilized at the UCSD Moores Cancer Center are the conical collimator on arc and the
Fig. 1 Patient undergoing a CT (FOV $35 \mathrm{~cm}, 1.25 \mathrm{~mm}$ slice thickness, no contrast) with mask and bite block in place

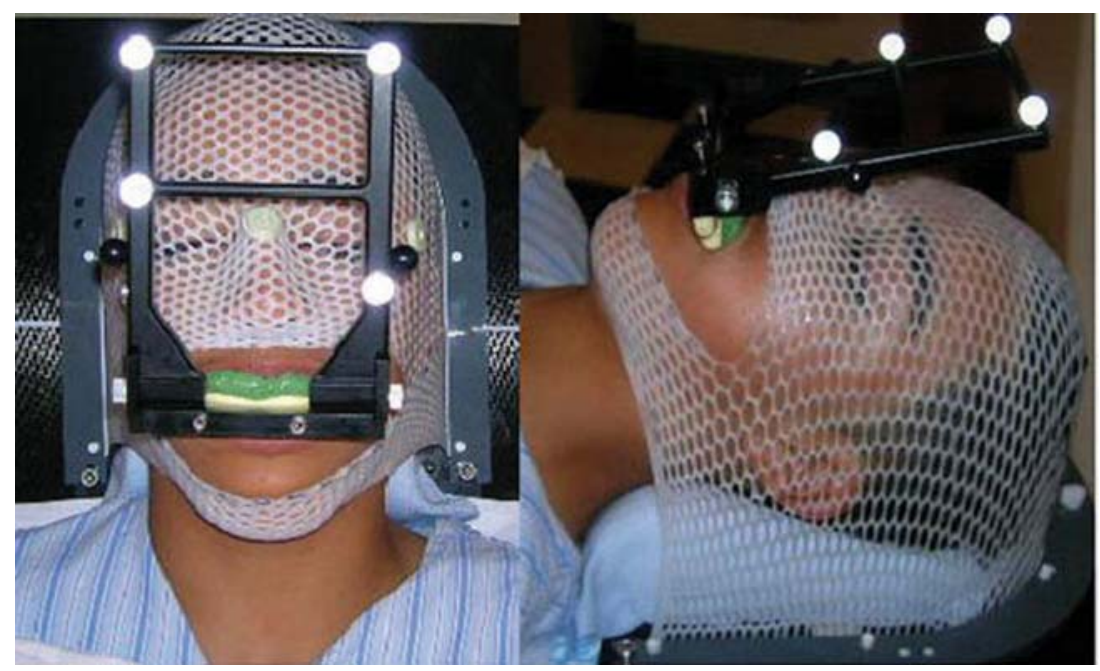




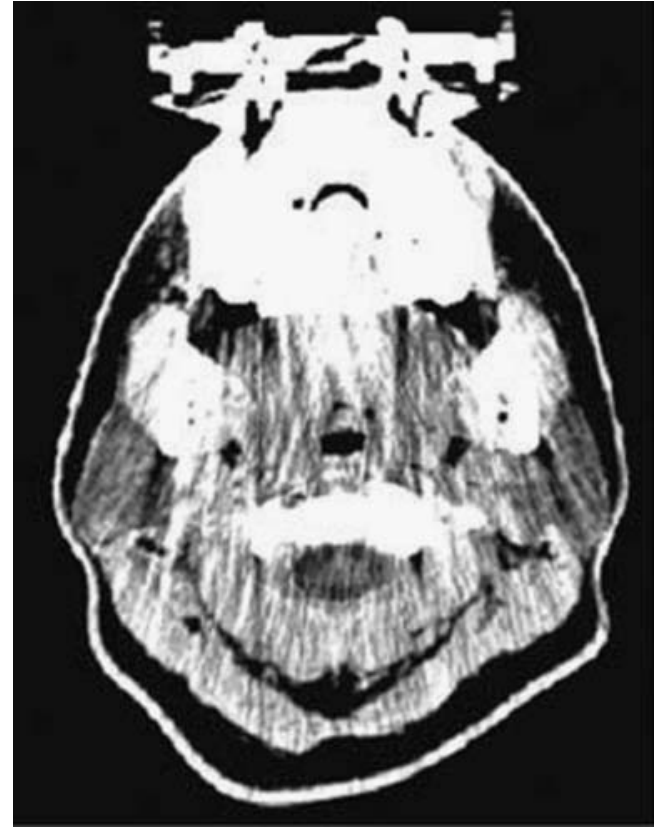

Fig. 2 With the CT registration, the Fastplan software detects, registers, and verifies fiducial markers

MLC with IMRs (Table 1). Standard arcs were selected from a database and the treatment dose and isodose lines were then delineated. Each fiducial on the bite block was digitized, with an average error in fiducial position of less than $0.75 \mathrm{~mm}$. The appropriate collimator (intensitymodulated radiosurgery with multi-leaf collimation or cone-based stereotactic radiosurgery) was attached and verified via the Winston-Lutz test, used for mechanical accuracy of the isocenter. Final adjustments were made using the optical array.

Throughout treatment, patients were observed via audio and visual monitors. Maintenance of patient positioning was assessed by the optical guidance camera. Our protocol is to interrupt treatment if there is a greater than $0.75 \mathrm{~mm}$ displacement of the reflective markers. This did not occur in this series, and all treatments were uninterrupted. Following completion of treatment, the mask, bite block, and array were removed. There were no specific treatmentrelated restrictions following completion of therapy.

\section{Treatments/results}

Out of an initial ten patients, nine were able to tolerate the procedure without requiring general anesthesia. Median age was 15 years (range $=12-19$ years). Two patients presented with juvenile pilocytic astrocytoma (JPA), one patient with a left pontine low-grade astrocytoma, three patients with pituitary adenoma, one patient with metastatic medullo- blastoma, one patient with bilateral acoustic schwannomas, and one patient with a pineocytoma (who had previously undergone optically guided frameless SRS and SRT; Table 1).

In all cases, the treatment was well tolerated with no acute toxicity requiring intervention. With a median followup of 12 months (range 3-18 months), no significant delayed toxicities have been observed. The radiographic imaging for Patients 1, 2, 5 and 7 are presented in Fig. 4-6.

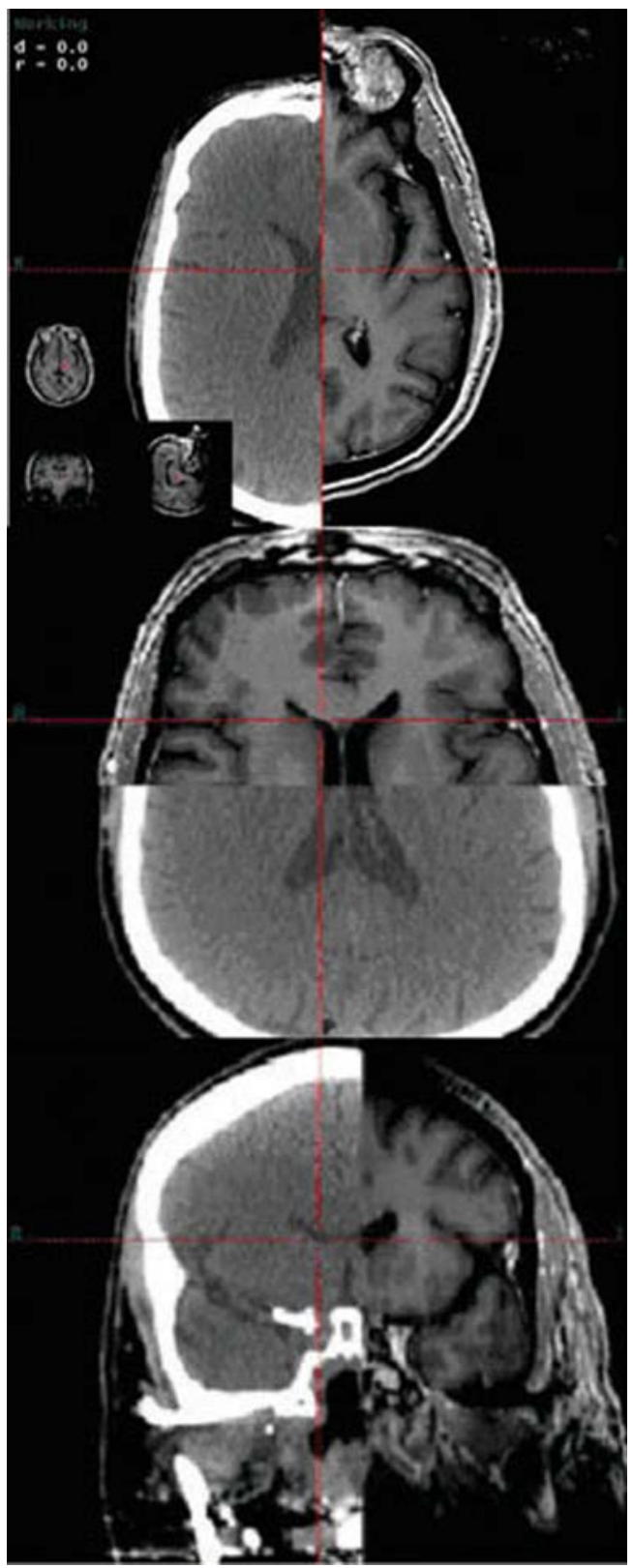

Fig. 3 The CT and MRI are manually fused, and then auto-fused 
Table 1 Patients, their diagnoses and treatment plans

\begin{tabular}{|c|c|c|c|c|c|c|c|}
\hline Patient & Diagnoses & Age & Treatment & $\begin{array}{l}\text { Type of } \\
\text { fractionation }\end{array}$ & Chemo & $\begin{array}{l}\text { Follow-up } \\
(\mathrm{mo})\end{array}$ & Response \\
\hline 1 & Low-grade astrocytoma & $16 \mathrm{Y}$ & $28 \times 1.8$ Gy & IMRS with MLC & Yes & 12 & Resolution \\
\hline 2 & $\begin{array}{l}\text { Juvenile Pilocytic } \\
\text { Astrocytoma }\end{array}$ & $12 \mathrm{Y}$ & $1 \times 20$ Gy & Conical Collimators & Yes & 6 & Resolution \\
\hline 3 & Pituitary Macroadenoma & $15 \mathrm{Y}$ & $28 \times 1.8$ Gy & IMRS with MLC & Yes & 12 & Required another resection \\
\hline 4 & Acoustic Schwannoma & $16 \mathrm{Y}$ & $28 \times 1.8$ Gy & IMRS with MLC & No & 3 & Stable \\
\hline 5 & Medulloblastoma Metastasis & $12 \mathrm{Y}$ & $14 \times 1$ Gy & Conical Collimators & Yes & 12 & Stable \\
\hline 6 & Pituitary Prolactinoma & $18 Y$ & $28 \times 1.8$ Gy & IMRS with MLC & Yes & 18 & Stable \\
\hline 7 & Pineocytoma & $19 \mathrm{Y}$ & $5 \times 5$ Gy & IMRS with MLC & No & 12 & Local control with subsequent metastasis \\
\hline 8 & $\begin{array}{l}\text { Juvenile Pilocytic } \\
\text { Astrocytoma }\end{array}$ & $14 \mathrm{Y}$ & $1 \times 20$ Gy & Conical Collimators & No & 10 & Stable \\
\hline 9 & Pituitary Macroadenoma & $12 \mathrm{Y}$ & $28 \times 1.8$ Gy & IMRS with MLC & Yes & 15 & Decreased GH level \\
\hline
\end{tabular}

Patient number 3: a 15-year-old female with a growth-hormone-secreting pituitary macroadenoma, underwent subtotal resection in December of 2005. Post-operatively had residual tumor in the right cavernous sinus that encased the cavernous portion of the carotid artery as well as the proximal right middle cerebral artery and anterior communicating artery. On serial MRI, she was noted to have subsequent tumor growth and marked mass effect on the optic chiasm. In August of 2006, she underwent fractionated radiotherapy to a dose of 50.4 Gy in 28 fractions of $1.8 \mathrm{~Gy}$. She tolerated the procedure well but on follow-up MRI, there was growth of her residual tumor, and she underwent a second resection in November of 2006

Patient number 4: a 16-year-old male with a history of type II neurofibromatosis with bilateral acoustic and trigeminal nerve neuromas. He received a course of fractionated IMRT to his left acoustic neuroma, which measured $2.5 \mathrm{~cm}$ in maximal diameter. He received a dose of $50.4 \mathrm{~Gy}$ in 28 fractions of $1.8 \mathrm{~Gy}$. Post-therapy, he was able to maintain hearing bilaterally without any new neurological deficits. There was no change in the size of his neuroma but there was some hypointensity in the central portion of the lesion suggestive of radiation therapy effect

Patient number 6: an 18-year-old male with a history of recurrent pituitary prolactinoma following multiple resections, most recently on December of 2005. Residual disease was noted involving the left cavernous sinus and associated left internal carotid artery. The patient continued to have elevated levels of prolactin despite aggressive endocrinologic intervention and his MRI was concerning for recurrence. He received a course of external beam stereotactic radiotherapy to his pituitary region to a dose of $50.4 \mathrm{~Gy}$, completed in April 2006. He tolerated the procedure and his existing bitemporal hemianopsia remains stable. He had no other neurological deficits. Follow-up MRI in November of 2006 indicated no increase in size of the pituitary mass with mild interval decrease in enhancement of the solid component, likely related to necrosis

Patient number 8: a 14-year-old male, post-two-surgical resections for a posterior fossa JPA with $1 \mathrm{~cm}$ residual adjacent to the site of the tumor resection. He underwent SRS to a dose of $20 \mathrm{~Gy}$. The patient tolerated the procedure well. At 10 months post-treatment, a follow-up MRI demonstrated regression of the lesion

Patient number 9: a 12-year-old male with a pituitary adenoma and acromegaly who underwent a resection in October of 2005. The patient had a residual intracavernous lesion measuring $1.4 \mathrm{~cm} \times 1.0 \mathrm{~cm}$ and received a dose of $50.4 \mathrm{~Gy}$ in May of 2006 with no complications. He has been continued on methimazole and somatostatin with reduction in his growth hormone levels. He suffered no focal neurological deficits and tolerated the procedure well

\section{Discussion}

In the pediatric population, there are typically significant concerns regarding treatment-related radiotoxicity, including potential neurocognitive and neuropsychological deficits, endocrine abnormalities, cerebral atrophy, skeletal bone growth delay/arrest, and secondary malignancy [11, 12]. Toxicity is known to be associated with the volume as well as nature of irradiated tissue. For example, reducing the amount of radiation exposure to normal brain parenchyma helps to minimize adverse treatment effects. Focal radiosurgery appears to have a better safety profile in preventing children's cognitive decline than whole-brain radiotherapy [12-17]. For such reasons, stereotactic radio- therapy is becoming a more integral part of brain tumor treatment in children.

Radiosurgery is able to deliver a high dose of radiation to a small target volume while providing relative sparing of surrounding normal tissue. The pediatric population presents unique difficulties in that general anesthesia and endotracheal intubation are often required to ensure compliance with head immobilization [18-20]. Other potential hazards related to head fixation include the risk of skull fracture with possible associated intracranial hemorrhage, especially in pediatric patients with thinner skulls and potential osteopenia secondary to past radiation treatments $[12,19]$. As a result, fractionated and hyperfractionated therapy with upward of 18-25 treatments can 

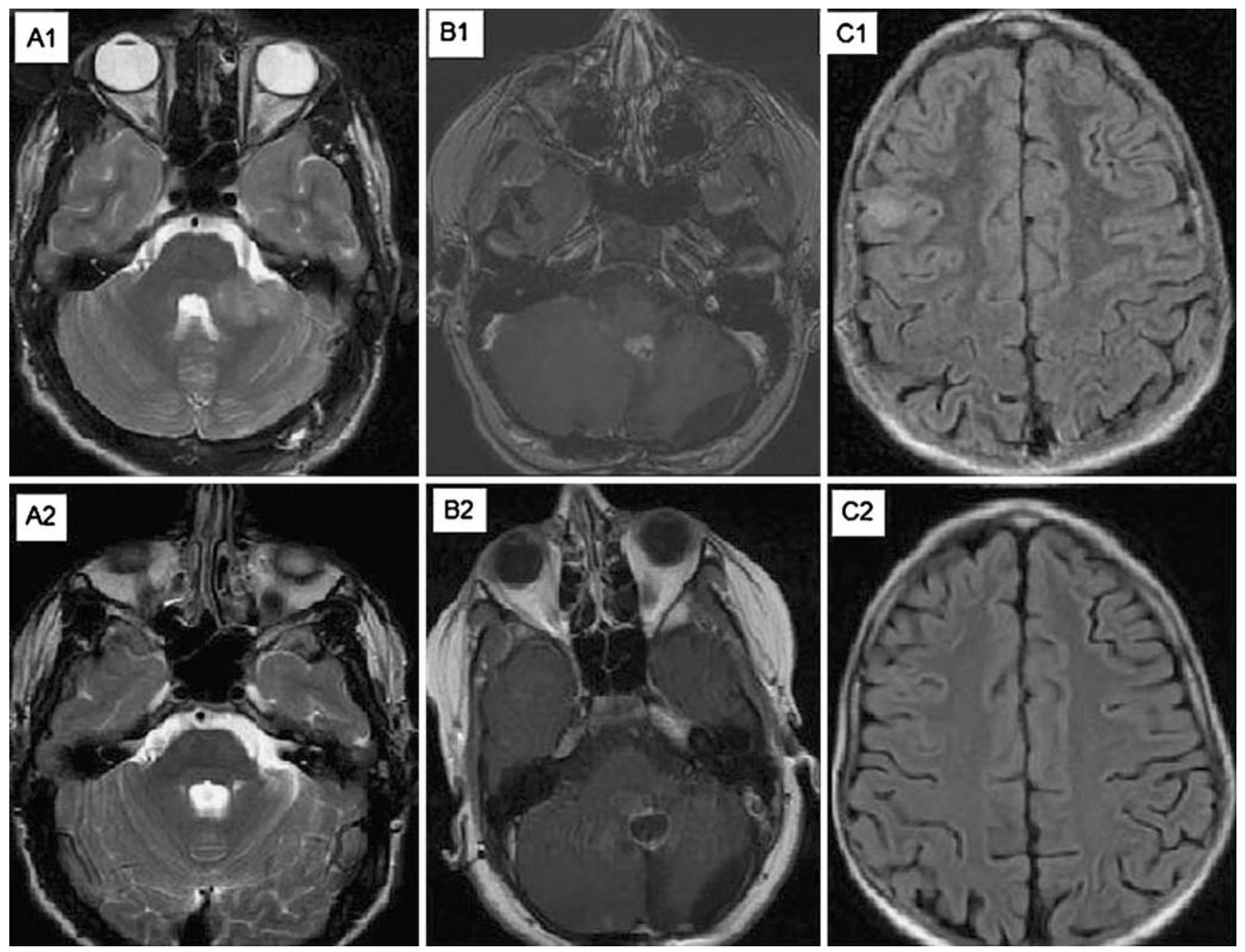

Fig. 4 a Patient 1 was a 16-year-old male with biopsy diagnosed left pontine low-grade astrocytoma $(A 1)$ underwent external beam radiotherapy to a dose of 5,400 cGy without complication (A2). He subsequently finished a course of Temodar in August of 2007. Prior to therapy, he was drooling and had a gait disturbance that has since resolved. He suffered from left-sided hearing loss, which has been stable since treatment and diplopia, corrected by glasses. b Patient 2 was a 12-year-old male diagnosed with juvenile pilocytic astrocytoma resected in 2001 and several years of chemotherapy (vincristine and carboplatin) completed in 2003. Follow-up imaging demonstrated a recurrence with a $10 \times 12 \mathrm{~mm}$ nodule along the anterior inferior fourth ventricle $(B 1)$. He underwent SRS in April of 2007. He tolerated the procedure well. Subsequent MRI demonstrated complete response, with slight residual enhancement in the surgical bed, with no nodularappearing enhancement (B2). c Patient 5 was a 12-year-old male diagnosed with medulloblastoma in 2003, underwent a gross-total resection with adjuvant chemotherapy and craniospinal radiotherapy. He received 36 Gy of craniospinal radiation with a posterior fossa boost in October of 2003 along with vincristine, completed in October of 2004. In June 2004, he was found to have recurrent cerebellar enhancement and diffuse leptomeningeal spread, and he was treated with temozolomide, etoposide, and cyclophosphamide. In 2006, MRI revealed an area of enhancement in the right frontoparietal region (C1), and he received radiosurgery with $14 \mathrm{~Gy}$, which the patient tolerated well. He now has local control at the frontoparietal site but continues to have leptomeningeal disease $(C 2)$ be challenging in this population. GammaKnife ${ }^{\circledR}$ has remained the gold standard for radiosurgical treatment for many years. GammaKnife ${ }^{\circledR}$ Radiosurgery (GKR) introduced by Lars Leksell in 1958 allows for accurate and focused targeting via the use of up to 201 individual radiation sources [21]. Treatment limitations in children are related to the duration of therapy, the use of rigid head fixation, and limitation to intracranial targets [12-17, 22,
23]. There has been a plethora of data utilizing GKR in children though a majority of the published experience details the treatment of arteriovenous malformations (AVM) $[13,14,24-27]$.

Advancements in motion management have allowed for the development of frameless techniques [12, 28, 29]. In adults, the use of frameless techniques has not been associated with any detriment to tumor control [10, 30- 
Fig. 5 Patient 7 was a 19-yearold female diagnosed with a large $(4.8 \mathrm{~cm} \times 4.0 \mathrm{~cm} \times 4.4 \mathrm{~cm})$ cystic lesion in her pineal region in July 2006. She underwent a subtotal resection revealing pineocytoma with increased mitotic activity. On her postoperative scan, a $15 \times 16-\mathrm{mm}$ enhancing nodule in the trigone of her left lateral ventricle and an $8 \times 6-\mathrm{mm}$ enhancing nodule in the floor of the third ventricle were discovered. She underwent a second surgical resection but continues to have residual tumor on post-operative MRI (a, b).

She received a fractionated course of radiosurgery to a dose of 2,500 cGy over five fractions, November 2006. She tolerated the procedure well and her pretreatment gait disturbance improved dramatically and on MRI of January $2007(\mathbf{c}, \mathbf{d})$
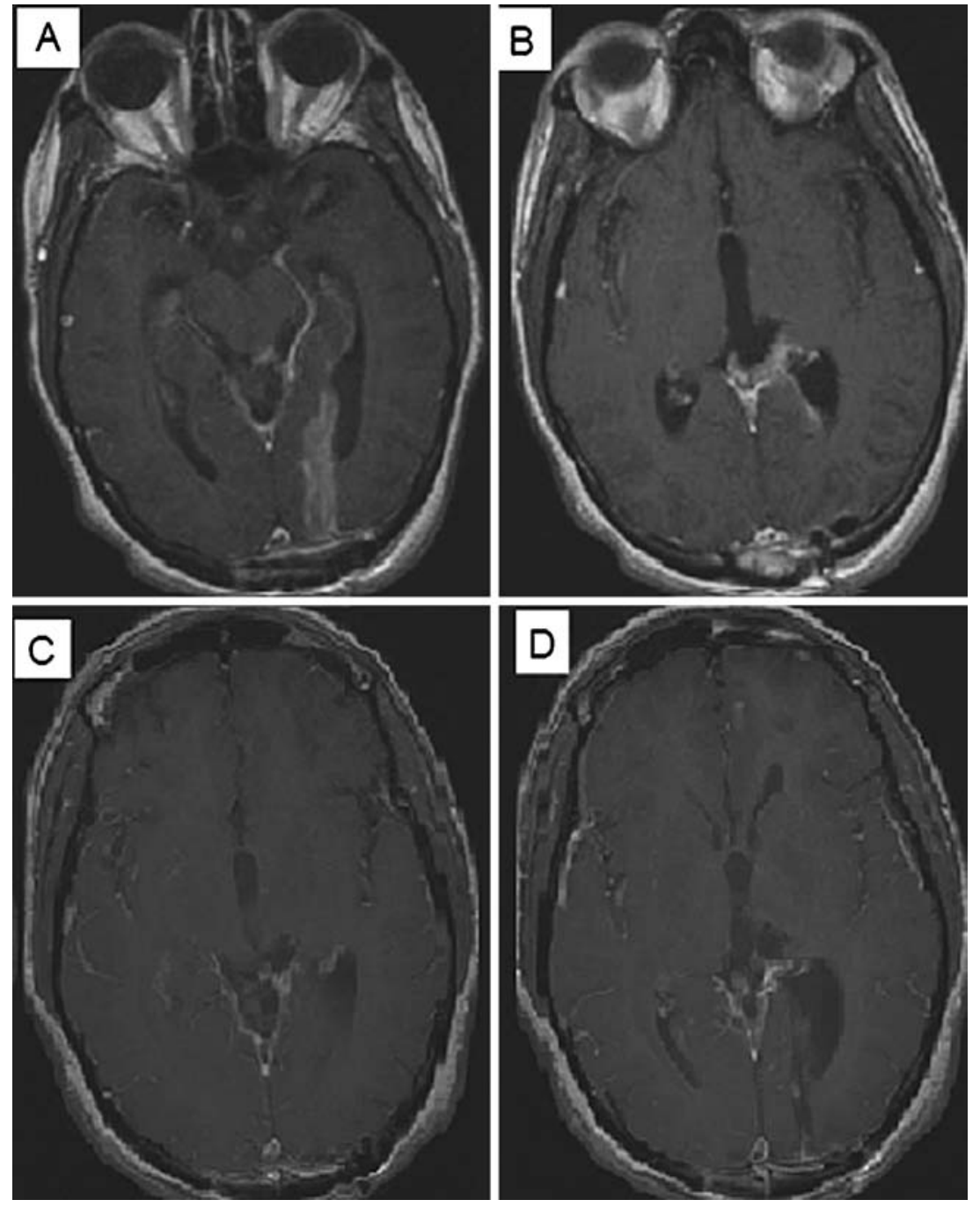

32]. Recently, several linear accelerator-based radiosurgical techniques have emerged including Cyberknife ${ }^{\circledR}$ (AccuraySunnyvale, CA), Trilogy (Varian Medical Systems- Palo Alto, CA), and Novalis (BrainLab AG) linear accelerator (LINAC) treatment systems [4, 33-37]. The Cyberknife ${ }^{\circledR}$ is a 6 MV LINAC mounted on a robotic arm, allowing for radiotherapy with the precision necessary to treat intracranial and extracranial tumors independent of frame-based rigid immobilization. Several series have been published detailing clinical experience in treating pediatric patients with this system $[12,38]$. Although Cyberknife ${ }^{\circledR}$ allows treatment of extracranial tumors and facilitates fractionated radiotherapy in the pediatric population, each treatment session generally lasts 60-90 min [39-41]. Large complex lesions may require treatment plans with multiple isocenters and increased treatment times which could require increased use of sedation in the pediatric population. Additionally, as opposed to the continuous monitoring of the optical guidance system described in this report, intrafraction motion management with Cyberknife is ac- complished using periodic X-ray images which results in additional patient exposure to non-therapeutic ionizing radiation $[12,38]$. Cyberknife ${ }^{\circledR}$ also does not allow for intensity modulation.

Trilogy and Novalis are LINAC systems that allow frameless targeting and single-fraction or fractionated treatment of intra- and extracranial lesions $[4,6,36]$. In addition to conical collimators, both Trilogy and Novalis utilize a multi-leaf collimator (MLC) with radiation treatment adapted to the shape of complex tumors, facilitating treatment and precluding the need for multiple isocenters. Other advantages include the use of intensity modulation to help limit toxicity to surrounding tissue [7] and treatments delivered in less than 30 min limiting the need for sedation. The frameless software used at UCSD allows for continuous patient monitoring utilizing infra-red optical guidance to track the patients' position during treatment delivery. Trilogy is novel in its use of optical guidance (non-ionizing radiation) for accuracy, sparing the patient additional radiation exposure. 

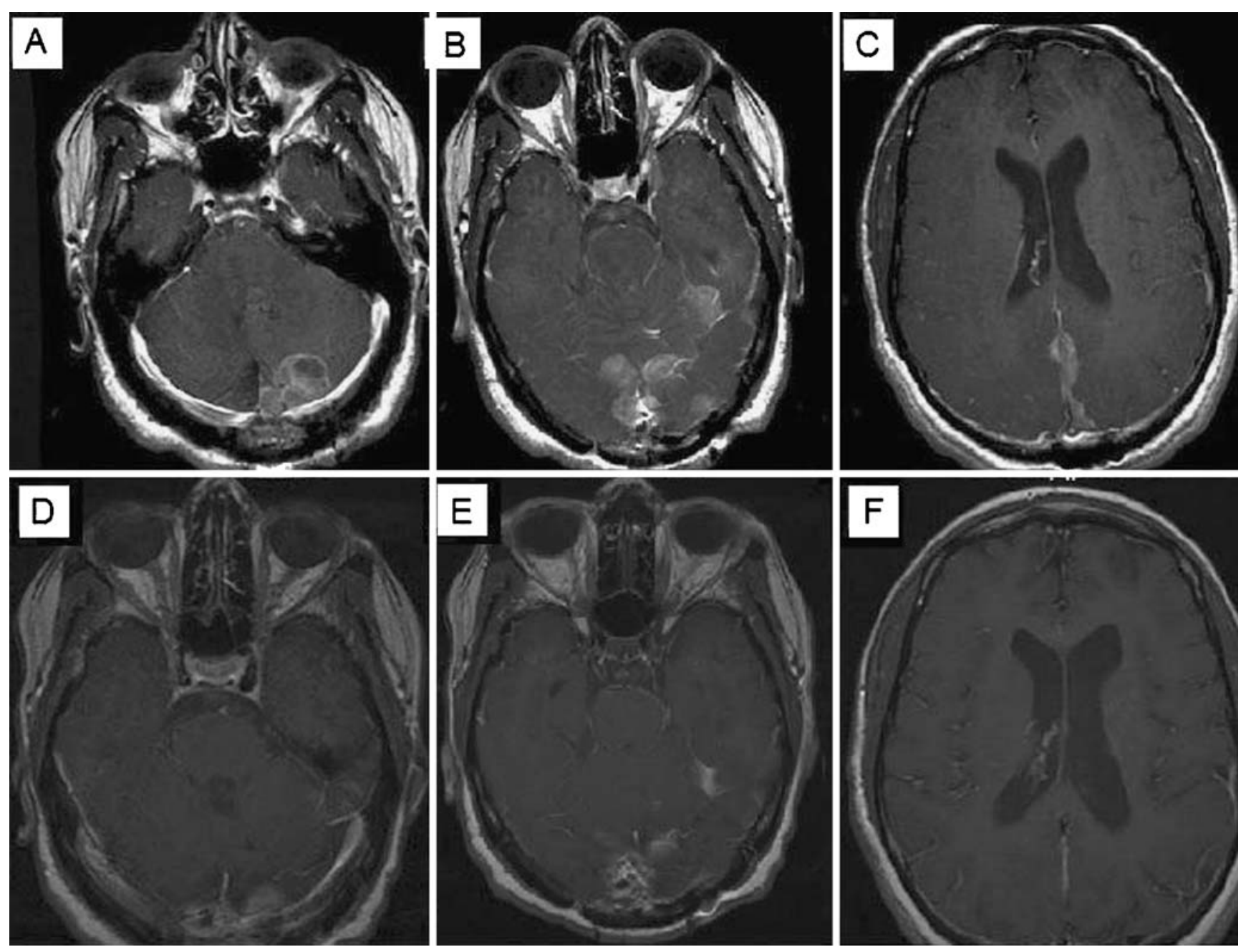

Fig. 6 On subsequent imaging of Patient 7 on November 2007, interval development of multiple enhancing dural masses involving the posterior falx, tentorium, posterior fossa, and the left middle cranial fossa have been discovered $(\mathbf{a}, \mathbf{b}, \mathbf{c})$. She subsequently received an additional 3060 cGy to her craniospinal axis March 2008. She has had marked improvement in her balance, coordination, and fine motor skills in both hands and improved on her subsequent MRI August 2008 (d, e, f)

\section{Conclusion}

In this retrospective study, we present our initial experience with pediatric and adolescent patients who underwent SRS and FSR with frameless optical guidance using the Trilogy linear accelerator system. There are several advantages to this system including the frameless technique, short beamon times, and continuous motion monitoring without the need for additional ionizing radiation exposure. No patients suffered treatment-related toxicities or developed new neurological deficits in follow-up and most patients either achieved stabilization or resolution of their tumors.

Conflict of Interest Dr. Kevin Murphy and the Department of Radiation Oncology at UCSD receives monetary support from Varian Corporation.

Open Access This article is distributed under the terms of the Creative Commons Attribution Noncommercial License which permits any noncommercial use, distribution, and reproduction in any medium, provided the original author(s) and source are credited.

\section{References}

1. Knab B, Connell PP (2007) Radiotherapy for pediatric brain tumors: when and how. Expert Rev Anticancer Ther 7(12):S69-S77

2. Robertson PL (2006) Advances in treatment of pediatric brain tumors. NeuroRX 3(2):276-291

3. Kortmann RD, Timmermann B, Taylor RE, Scarzello G, Plasswilm L, Paulsen F, Jeremic B, Gnekow AK, Dieckmann K, Kay S, Bamberg M (2003) Current and future strategies in radiotherapy of childhood low-grade glioma of the brain. Part II: treatment-related late toxicity. Strahlenther Onkol 179(9):585597 
4. Chen JC, Rahimian J, Girvigian MR, Miller MJ (2007) Contemporary methods of radiosurgery treatment with the Novalis linear accelerator system. Neurosurg Focus 23(6):E4

5. Chen CC, Chapman P, Petit J, Loeffler J (2007) Proton radiosurgery in neurosurgery. Neurosurg Focus 23(6):1-5

6. Yin FF, Zhu J, Yan H, Gaun H, Hammoud R, Ryu S, Kim JH (2002) Dosimetric characteristics of Novalis shaped beam surgery unit. Med Phys 29(8):1729-1738

7. Wiersma RD, Xing L (2007) Examination of geometric and dosimetric accuracies of gated step-and-shoot intensity modulated radiation therapy. Med Phys 34(10):3962-3970

8. Chang SD, Main W, Martin DP, Gibbs IC, Heilbrun MP (2003) An analysis of the accuracy of the CyberKnife: a robotic frameless stereotactic radiosurgical system. Neurosurgery 52(1):140-146. discussion 146-147

9. Gerszten PC, Ozhasoglu C, Burton SA, Vogel WJ, Atkins BA, Kalnicki S, Welch WC (2004) CyberKnife frameless stereotactic radiosurgery for spinal lesions: clinical experience in 125 cases. Neurosurgery 55(1):89-98. discussion 98-99

10. Steffey-Stacy EC (2006) Frameless, image-guided stereotactic radiosurgery. Sem Oncol Nurs 22(4):221-232

11. Inoue HK, Kohga H, Nakamura M, Ono N, Kakegawa T, Hirato M, Ohye C, Shibazaki T, Andou Y (1994) Long-term follow-up study of conventional irradiation for brain tumours in children: a role for radiosurgery. Acta neurochirurgica 62:83-87

12. Giller CA, Berger BD, Pistenmaa DA, Sklar F, Weprin B, Shapiro K, Winick N, Mulne AF, Delp JL, Gilio JP, Gall KP, Dicke KA, Swift D, Sacco D, Harris-Henderson K, Bowers D (2005) Robotically guided radiosurgery for children. Pediatr Blood Cancer 45(3):304-310

13. Baumann GS, Wara WM, Larson DA, Sneed PK, Gutin PH, Ciricillo SF, McDermott MW, Park E, Stalpers LJ, Verhey LJ, Smith V, Petti PL, Edwards MS (1996) Gamma knife radiosurgery in children. Pediatr Neurosur 24(4):193-201

14. Eder HG, Leber KA, Eustacchio S, Pendl G (2001) The role of gamma knife radiosurgery in children. Childs Nerv Syst 17 (6):341-346. discussion 7

15. Gerszten PC, Adelson PD, Kondziolka D, Flickinger JC, Lunsford LD (1996) Seizure outcome in children treated for arteriovenous malformations using gamma knife radiosurgery. Pediatr Neurosurg 24:139-144

16. Grabb PA, Lunsford LD, Albright AL, Kondziolka D, Flickinger JC (1996) Stereotactic radiosurgery for glial neoplasms of childhood. Neurosurgery 38:696-702

17. Kondziolka D, Lunsford LD, Flickinger JC (1991) Stereotactic radiosurgery in children and adolescents. Pediatr Neurosurg 16:219-221

18. Baker KC, Isert PR (1997) Anaesthetic considerations for children undergoing stereotactic radiosurgery. Anaesth Intens Care 25:691-695

19. Elder A (2007) Special anesthetic considerations for stereotactic radiosurgery in children. J Clin Anesth 19:616-618

20. Stokes MA, Soriano SG, Tarbell NJ, Loeffler JS, Alexander E 3 rd, Black PM, Rockoff MA (1995) Anesthesia for stereotactic radiosurgery in children. J Neurosurg Anesthesiol 7:100-108

21. Larsson B, Leksell L, Rexed B, Sourander P, Mair W, Andersson B (1958) The high-energy proton beam as a neurosurgical tool. Nature 182(4644):1222-1223

22. Lindquist C, Paddick I (2007) The Leksell Gamma Knife Perfexion and comparisons with its predecessors. Neurosurgery 61(3 Suppl):130-140. discussion 40-41

23. Weprin BE, Hall WA, Cho KH, Sperduto PW, Gerbi BJ, Moertel C (1996) Stereotactic radiosurgery in pediatric patients. Pediatr Neurol 15:193-199

24. Pan DH, Kuo YH, Guo WY, Chung WY, Wu HM, Liu KD, Chang YC, Wang LW, Wong TT (2008) Gamma Knife surgery for cerebral arteriovenous malformations in children: a 13-year experience. J Neurosurg 1(4):296-304
25. Nicolato A, Lupidi F, Sandri MF, Foroni R, Zampieri P, Mazza C, Pasqualin A, Beltramello A, Gerosa M (2006) Gamma Knife radiosurgery for cerebral arteriovenous malformations in children/ adolescents and adults. Part II: Differences in obliteration rates, treatment-obliteration intervals, and prognostic factors. Int $\mathrm{J}$ Radiat Oncol, Biol, Phys 64(3):914-921

26. Nicolato A, Foroni R, Seghedoni A, Martines V, Lupidi F, Zampieri P, Sandri MF, Ricci U, Mazza C, Beltramello A, Gerosa M, Bricolo A (2005) Leksell gamma knife radiosurgery for cerebral arteriovenous malformations in pediatric patients. Childs Nerv Syst 21(4):301-307. discussion 8

27. Yamamoto M (1999) Gamma Knife radiosurgery: technology, applications, and future directions. Neurosurg Clin N Am 10 (2): 181-202

28. Bova FJ, Buatti JM, Friedman WA, Mendenhall WM, Yang CC, Liu C (1997) The University of Florida frameless high-precision stereotactic radiotherapy system. Int J Radiat Oncol, Biol, Phys 38 (4):875-882

29. Solberg TD, Medin PM, Mullins J, Li S (2008) Quality assurance of immobilization and target localization systems for frameless stereotactic cranial and extracranial hypofractionated radiotherapy. Int J Radiat Oncol, Biol, Phys 71(1 Suppl):S131-S135

30. Gibbs IC (2006) Frameless image-guided intracranial and extracranial radiosurgery using the Cyberknife robotic system. Cancer Radiother 10(5):283-287

31. Kamath R, Ryken TC, Meeks SL, Pennington EC, Ritchie J, Buatti JM (2005) Initial clinical experience with frameless radiosurgery for patients with intracranial metastases. Int J Radiat Oncol, Biol, Phys 61(5):1467-1472

32. Ryken TC, Meeks SL, Pennington EC, Hitchon P, Traynelis V, Mayr NA, Bova FJ, Friedman WA, Buatti JM (2001) Initial clinical experience with frameless stereotactic radiosurgery: analysis of accuracy and feasibility. Int J Radiat Oncol, Biol, Phys 51(4):1152-1158

33. Alexander E 3 rd, Loeffler JS (1992) Radiosurgery using a modified linear accelerator. Neurosurg Clin N Am 3(1):167-190

34. Hara W, Soltys SG, Gibbs IC (2007) CyberKnife robotic radiosurgery system for tumor treatment. Expert Review of Anticancer Therapy 7(11):1507-1515

35. Hitchcock E, Kitchen G, Dalton E, Pope B (1989) Stereotactic LINAC radiosurgery. Br J Neurosurg 3(3):305-312

36. Huntzinger C, Friedman W, Bova F, Fox T, Bouchet L, Boeh L (2007) Trilogy image-guided stereotactic radiosurgery. Med Dosim 32(2):121-133

37. Mitsumori M, Shrieve DC, Alexander E 3 rd, Kaiser UB, Richardson GE, Black PM, Loeffler JS (1998) Initial clinical results of LINAC-based stereotactic radiosurgery and stereotactic radiotherapy for pituitary adenomas. Int J Radiat Oncol, Biol, Phys 42(3):573-580

38. Giller CA, Berger BD, Gilio JP, Delp JL, Gall KP, Weprin B, Bowers $D$ (2004) Feasibility of radiosurgery for malignant brain tumors in infants by use of image-guided robotic radiosurgery: preliminary report. Neurosurgery 55(4):916-924. discussion 924-925

39. Brown WT, Wu X, Amendola B, Perman M, Han H, Fayad F, Garcia S, Lewin A, Abitbol A, de la Zerda A, Schawade JG (2007) Treatment of early non-small cell lung cancer, stage IA, by image-guided robotic stereotactic radioablation - CyberKnife. Cancer J 13(2):87-94

40. Brown WT, Wu X, Fayad F, Fowler JF, Amendola BE, Garcia S, Han H, de la Zerda A, Bossart E, Huang Z, Schwade JG (2007) CyberKnife radiosurgery for stage I lung cancer: results at 36 months. Clin Lung Cancer 8(8):488-492

41. Villavicencio AT, Lim M, Burneikiene S, Romanelli P, Adler JR, McNeely L, Chang SD, Fariselli L, McIntyre M, Bower R, Broggi G, Thramann JJ (2008) Cyberknife radiosurgery for trigeminal neuralgia treatment: a preliminary multicenter experience. Neurosurgery 62(3):647-655. discussion -55 